\title{
Survie des bactéries lactiques soumises à la déshydratation : importance de la cinétique de déshydratation
}

\author{
Isabelle Poirier, Pierre-André Marechal, Patrick Gervais*
}

Laboratoire de génie des procédés alimentaires et biotechnologiques, Ensbana, 1, esplanade Érasme, 21000 Dijon, France

\begin{abstract}
Viability of lactic bacteria submitted to dehydration: Importance of the dehydration kinetics. Dehydration of Lactobacillus plantarum by glycerol addition (water depressor) has allowed to show the great importance of the kinetics of water potential decrease on cell viability. Thus, wathever the final water potential was, an optimal kinetics of water potential variation was determined which allow to maintain a viability higher than $80 \%$. Decreasing rate and level of water potential have been proved to influence cell viability in the same way during air drying at $25^{\circ} \mathrm{C}$. In this case, optimal kinetics of dehydration preserved $40 \%$ of viable cells. This viability was higher than $80 \%$ when a nutritional stress was realised before dehydration and when cells were rehydrated at $37^{\circ} \mathrm{C}$. A high preservation of dehydrated cells was observed for 3 months at $-317.9 \mathrm{MPa}\left(\mathrm{a}_{\mathrm{w}}=0.100\right)$; and after rehydration, stressed cells exhibited an acid production similar to a control. (C) Inra/Elsevier, Paris.
\end{abstract}

\section{Lactobacillus plantarum / dehydration / drying / kinetics}

Résumé - La déshydratation de Lactobacillus plantarum par l'addition de glycérol (soluté dépresseur de l'activité de l'eau) a mis en évidence l'importance de la cinétique de diminution du potentiel hydrique sur la viabilité cellulaire. Pour chaque niveau de potentiel hydrique atteint, une cinétique optimale de diminution du potentiel hydrique a été déterminée, permettant de maintenir plus de $80 \%$ de cellules viables. Le même phénomène a été observé lors de la déshydratation de cette espèce bactérienne par séchage par air sec à $25^{\circ} \mathrm{C}$, où une cinétique optimale de déshydratation a permis de maintenir $40 \%$ de cellules viables. Ce taux de viabilité est supérieur à $80 \%$ lorsqu'un stress nutritionnel est réalisé avant déshydratation et lorsque la réhydratation est effectuée à $37^{\circ} \mathrm{C}$. Les cellules déshydratées à un potentiel hydrique de $-317,9 \mathrm{MPa}\left(\mathrm{a}_{\mathrm{w}}=0,100\right)$ présentent une bonne conservation sur trois mois, et montrent après réhydratation, une activité acidifiante comparable à celle de bactéries non traitées. (C) Inra/Elsevier, Paris.

Lactobacillus plantarum / déshydratation / séchage / cinétique

* Correspondance et tirés à part 


\section{INTRODUCTION}

La conservation des microorganismes représente un enjeu économique majeur dans l'industrie agro-alimentaire. Les techniques de conservation les plus couramment employées actuellement sont les techniques faisant appel à la déshydratation, tels que la congélation, la lyophilisation ou le séchage. Déshydrater une suspension bactérienne revient à diminuer le potentiel hydrique $(\psi)$ de cette suspension, ainsi que son activité de l'eau $\left(\mathrm{a}_{\mathrm{w}}\right)$ puisque ces deux derniers paramêtres sont directement liés par l'équation (1) suivante :

$$
\psi(\mathrm{Pa})=\frac{+ \text { RTlna }_{\mathrm{w}}}{\mathrm{V}_{\mathrm{w}}}
$$

où $\mathrm{R}=$ cte des gaz parfaits $\left(8,31 \mathrm{~J} \mathrm{~mol}^{-1}\right.$ $\left.\mathrm{K}^{-1}\right), \mathrm{T}=$ température $(298 \mathrm{~K})$ et $\mathrm{V}_{\mathrm{w}}=$ volume molaire partiel de l'eau $(1,8$, $\left.10^{-5} \mathrm{~m}^{3} \mathrm{~mol}^{-1}\right)$.

$L^{\prime} \mathrm{a}_{\mathrm{w}}$ est un facteur très important qui agit sur la physiologie et le métabolisme des microorganismes [2].

Les différents procédés de déshydratation existants peuvent entraîner des dommages cellulaires importants tels que la fragmentation du système vacuolaire, la condensation de la molécule d'ADN ou une modification de la solubilité de certaines protéines [1]. Ces modifications entrainnent des taux de mortalité élevés.

Toutefois, au cours de ces différents procédés de déshydratation, la viabilité cellulaire peut être préservée par l'addition d'osmoprotectants dans le milieu de déshydratation [5]. D'autres études ont mis en évidence l'importance de la température, de la cinétique de réhydratation [9], ainsi que de la cinétique de déshydratation [4] sur la viabilité cellulaire.

Nous avons donc étudié l'influence de ces différents paramètres sur la viabilité de Lactobacillus plantarum lors de procédés de déshydratation, afin d'optimiser les conditions de conservation des microorganismes pour une utilisation industrielle.

\section{MATÉRIEL ET MÉTHODES}

\subsection{Microorganismes et conditions de culture}

Une souche bactérienne a été utilisée pour cette étude : Lactobacillus plantarum souche L-73 (CHR Hansen, St-Germain-les-Arpajon, France).

Cette souche bactérienne est conservée à $4{ }^{\circ} \mathrm{C}$ sur gélose MRS [3]. Le potentiel hydrique initial de ce milieu est fixé à $-1,4 \mathrm{MPa}$ $\left(\mathrm{a}_{\mathrm{w}}=0,990\right)$ par l'addition de glycérol $(52 \mathrm{~g} / \mathrm{L})$.

Pour chaque expérience, les cultures sont réalisées en milieu MRS, inoculé à partir de $0,1 \mathrm{~mL}$ d'une préculture de 12 heures. Les cultures sont utilisées à la fin de la phase exponentielle de croissance.

\subsection{Déshydratation en milieu liquide}

\subsubsection{Influence de la cinétique de déshydratation}

La déshydratation en milieu liquide par 1'addition de glycérol (soluté dépresseur de l'activité de l'eau) est réalisée selon le protocole décrit par Poirier et al. [8].

L. plantarum a été soumise à trois niveaux de potentiel hydrique : $-107,2 \mathrm{MPa}\left(\mathrm{a}_{\mathrm{w}}=0,460\right)$, $-170,9 \mathrm{MPa}\left(\mathrm{a}_{\mathrm{w}}=0,290\right)$ et $-244,7 \mathrm{MPa}$ $\left(a_{w}=0,170\right)$; et pour chaque niveau le glycérol est ajouté selon différents débits afin d'engendrer différentes cinétiques de déshydratation. De plus, un traitement correspondant à un mélange rapide du glycérol et du culot bactérien est réalisé et assimilé à un choc osmotique.

\subsubsection{Mesure de la viabilité cellulaire}

La mesure de la viabilité cellulaire est réalisée par la méthode UFC, comme décrit par Poirier et al. [8]. 


\subsection{Déshydratation par séchage}

\subsubsection{Influence de la cinétique de déshydratation}

La déshydratation par séchage est réalisée en étuve MPC (Neuilly-sur-Marne, France) pour des cinétiques de déshydratation d'une durée supérieure à 3 heures; et en dessiccateur pour des cinétiques de déshydratation d'une durée inférieure à 3 heures. L'étuve MPC est une étuve industrielle où l'air est ventilé. A l'intérieur de l'enceinte, il est possible de faire varier la température et l'humidité relative de l'air afin d'obtenir différentes cinétiques de déshydratation. La déshydratation en dessiccateur consiste à injecter, à l'intérieur de l'enceinte où sont placés les échantillons, de l'air sec à différents débits.

Après 24 heures de culture en milieu MRS, $100 \mathrm{~mL}$ de la suspension bactérienne sont centrifugés ( 5 min à $2860 \mathrm{~g}$ ). Le culot cellulaire est ajouté à $60 \mathrm{~g}$ de farine et à $140 \mathrm{~mL}$ d'eau stérile. Ce mélange est ensuite réparti en boîtes de Pétri à raison de $5 \mathrm{~mL}$ par boîte. Les boîtes de Pétri sont alors placées dans l'étuve MPC, ou en dessiccateur sans couvercle pour réaliser les différentes cinétiques de déshydratation souhaitées. La température de déshydratation a été fixée à $25^{\circ} \mathrm{C}$.

Au cours de ce séchage des prélèvements sont effectués afin de contrôler l'évolution du potentiel hydrique des échantillons. Deux potentiels hydriques finaux ont été atteints : $-166,2 \mathrm{MPa}\left(\mathrm{a}_{\mathrm{w}}=0,300\right)$ et $-317,9 \mathrm{MPa}\left(\mathrm{a}_{\mathrm{w}}\right.$ $=0,100)$.

Trois cinétiques de déshydratation ont été testées en étuve MPC : $-0,0019 \mathrm{MPa} . \mathrm{s}^{-1}$, $-0,0076 \mathrm{MPa} . \mathrm{s}^{-1},-0,0102 \mathrm{MPa} . \mathrm{s}^{-1}$; et deux en dessiccateur : $-0,0229 \mathrm{MPa}^{-1},-0,0763 \mathrm{MPa}^{-1} \mathrm{~s}^{-1}$.

\subsubsection{Influence de la température de réhydratation}

Un gramme des échantillons déshydratés selon des cinétiques de déshydratation de $-0,0229 \mathrm{MPa} . \mathrm{s}^{-1}$ et $-0,0480 \mathrm{MPa} . \mathrm{s}^{-1}$ afin d'obtenir respectivement des potentiels hydriques finaux de $-166,2 \mathrm{MPa}\left(\mathrm{a}_{\mathrm{w}}=0,300\right)$ et $-317,9 \mathrm{MPa}\left(\mathrm{a}_{\mathrm{w}}=0,100\right)$ est réhydraté un quart d'heure en milieu MRS à deux températures : $25^{\circ} \mathrm{C}$ et $37^{\circ} \mathrm{C}$.

\subsubsection{Préparation des cellules à subir la déshydratation}

Parallèlement aux expériences décrites précédemment, le culot bactérien est repris dans $50 \mathrm{~mL}$ d'une solution binaire eau-glycérol $(\psi=-1,4 \mathrm{MPa})$. L'ensemble est laissé à $25^{\circ} \mathrm{C}$ pendant $1 / 4$ d'heure (stress nutritionnel) avant d'être mélangé à $15 \mathrm{~g}$ de farine. Ce mélange est alors réparti en boîtes de Pétri, à raison de $5 \mathrm{~mL}$ par boîte et est placé en dessiccateur pour subir des cinétiques de déshydratation de $-0,0229 \mathrm{MPa} \cdot \mathrm{s}^{-1}$ et $-0,0480 \mathrm{MPa} \cdot \mathrm{s}^{-1}$ afin d'obtenir respectivement des potentiels hydriques finaux de $-166,2 \mathrm{MPa}\left(\mathrm{a}_{\mathrm{w}}=0,300\right)$ et $-317,9 \mathrm{MPa}\left(\mathrm{a}_{\mathrm{w}}=0,100\right)$. Les échantillons obtenus sont réhydratés un quart d'heure en milieu MRS à $37^{\circ} \mathrm{C}$ pour mesurer la viabilité cellulaire,

\subsubsection{Mesure de la viabilité cellulaire}

Un gramme du produit déshydraté est réhydraté en milieu MRS à $25^{\circ} \mathrm{C}$ afin de mesurer la viabilité cellulaire par UFC par comparaison à un témoin réalisé avant déshydratation.

\subsubsection{Mesure de l'activité acidifiante de L. plantarum après réhydratation à $37^{\circ} \mathrm{C}$ en milieu MRS}

La reprise d'activité métabolique des cellules déshydratées par séchage à un potentiel hydrique de $-317,9 \mathrm{MPa}(\mathrm{aw}=0,100)$ est mesurée par le suivi de la cinétique d'acidification du milieu MRS en fermenteur à $30^{\circ} \mathrm{C}$. Cette cinétique est comparée à celle du témoin non déshydraté et à celle de la même souche lyophilisếe. Les fermenteurs sont reliés à un micro-ordinateur qui contrôle la régulation de la température. Toutes les 10 minutes, le $\mathrm{pH}$ est mesuré par l'intermédiaire d'une sonde $\mathrm{pH}$ et est automatiquement enregistré.

\subsection{6. Étude de conservation des échantillons déshydratés}

Les échantillons déshydratés sont fractionnés et conditionnés sous azote. Les sachets sont ensuite placés à $4{ }^{\circ} \mathrm{C}$ à l'obscurité. Au temps $t=0$ après déshydratation, puis à différents temps pendant 3 mois, le contenu d'un sachet est réhydraté afin de suivre l'évolution de la viabilité cellulaire. 


\section{RÉSULTATS}

\subsection{Déshydratation en milieu liquide}

Lorsque $L$. plantarum est soumis à un potentiel hydrique de $-170,9 \mathrm{MPa}$ et $-244,7 \mathrm{MPa}$, la viabilité dépend de la cinétique de diminution du potentiel hydrique (tableau I). La viabilité cellulaire après un choc osmotique de $-170,9 \mathrm{MPa}$ et $-244,7 \mathrm{MPa}$ est respectivement de $41 \%$ et $15 \%$, alors que des diminutions lentes du potentiel hydrique permettent de maintenir $85 \%$ des cellules viables. De plus, lorsque cette espèce bactérienne est soumise à un potentiel hydrique de $-244,7 \mathrm{MPa}$, une cinétique optimale de diminution du potentiel hydrique a été mise en évidence. En revanche, lorsque cette espèce bactérienne est soumise à un potentiel hydrique de $-107,2 \mathrm{MPa}$, la viabilité n'est pas significativement différente entre une diminution rapide et une diminution lente du potentiel hydrique.

\subsection{Déshydratation par séchage}

\subsubsection{Influence de la cinétique de déshydratation sur la viabilité cellulaire}

Les résultats présentés dans le tableau II montrent également l'importance de la cinétique de déshydratation sur la viabilité cellulaire. Pour chaque potentiel hydrique testé, une cinétique optimale de déshydratation a été mise en évidence permettant de maintenir plus de $35 \%$ de viabilité. Ces cinétiques sont proches de celles déterminées en milieu liquide.

\subsubsection{Influence de la température de réhydratation}

Les résultats présentés dans le tableau III montrent l'importance de la température de réhydratation sur la viabilité bactérienne. Une réhydratation à $37^{\circ} \mathrm{C}$ permet de maintenir viables $60 \%$ des cellules pour les deux niveaux de potentiel

Tableau I. Viabilité (\%) de L. plantarum en fonction de la cinétique de diminution du potentiel hydrique. Déshydratation en milieu liquide par addition de glycérol. Potentiel hydrique initial = $-1,4 \mathrm{MPa}$; dépresseur de l'eau utilisé : glycérol.

Table I. Effect of the kinetics of water potential decrease on L. plantarum viability (\%). Dehydration is realised in liquid medium by glycerol addition. Initial water potential: $-1.4 \mathrm{MPa}$. Water depressor used: glycerol.

\begin{tabular}{ccrc}
\hline $\begin{array}{c}\text { Potentiel hydrique final } \\
(\mathrm{MPa}) \\
\left(\mathrm{a}_{\mathrm{w}} \text { finale }\right)\end{array}$ & $\begin{array}{c}\text { Cinétique de variation du } \\
\text { potentiel hydrique }\left(\mathrm{MPa}^{-1}\right)\end{array}$ & $\begin{array}{c}\text { Durée } \\
(\mathrm{min})\end{array}$ & $\begin{array}{c}\text { Viabilité } \\
(\% \text { par rapport au témoin })\end{array}$ \\
\hline & $-105,8$ & shock & $76,6 \pm 5,8$ \\
$-107,2$ & $-0,025$ & 70 & $73,4 \pm 5,5$ \\
$(0,460)$ & $-0,008$ & 230 & $85,4 \pm 6,4$ \\
& $-169,52$ & shock & $41,0 \pm 1,7$ \\
$-170,9$ & $-0,257$ & 11 & $76,3 \pm 2,9$ \\
$(0,290)$ & $-0,118$ & 24 & $88,0 \pm 2,7$ \\
& $-0,057$ & 50 & $90,3 \pm 2,5$ \\
& $-0,021$ & 135 & $85,3 \pm 2,5$ \\
& $-243,32$ & shock & $15,3 \pm 2,1$ \\
$-244,7$ & $-0,088$ & 46 & $39,5 \pm 0,7$ \\
$(0,170)$ & $-0,061$ & 66 & $81,2 \pm 7,0$ \\
& $-0,037$ & 110 & $85,8 \pm 5,4$ \\
& $-0,025$ & 160 & $51,3 \pm 5,0$ \\
& $-0,015$ & 270 & $26,5 \pm 0,7$
\end{tabular}


Tableau II. Viabilité (\%) de L. plantarum en fonction de la cinétique de diminution du potentiel hydrique. Déshydratation par séchage en milieu farine eau. Potentiel hydrique initial $=-1,4$ $\mathrm{MPa}$.

Table II. Effect of the kinetics of water potential decrease on L. plantarum viability (\%). Dehydration is realised by drying in a flour-water mixture. Initial water potential: $-1.4 \mathrm{MPa}$.

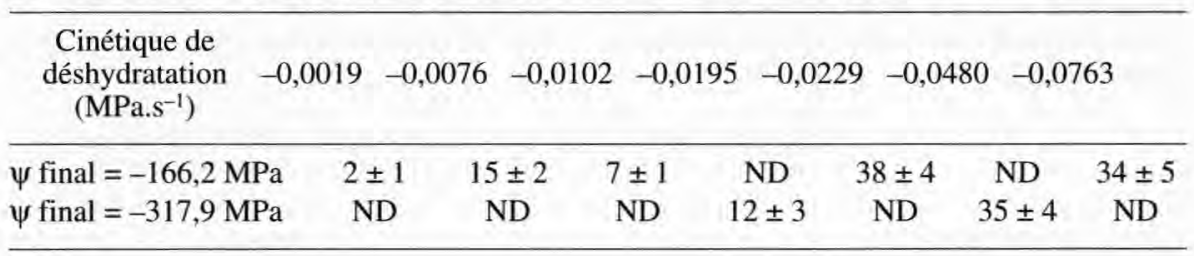

Tableau III. Viabilité (\%) de $L$. plantarum en fonction de la température de réhydratation. Déshydratation par séchage en milieu farine eau selon des cinétiques de déshydratation de $-0,0229 \mathrm{MPa} . \mathrm{s}^{-1}$ et $-0,0480 \mathrm{MPa} \cdot \mathrm{s}^{-1}$ afin d'obtenir respectivement des potentiels hydriques finaux $\mathrm{de}-166,2 \mathrm{MPa}$ et $-317,9 \mathrm{MPa}$. Potentiel hydrique initial $=-1,4 \mathrm{MPa}$.

Table III. Effect of the rehydration temperature on L. plantarum viability (\%). Dehydration is realised by drying in a flour-water mixture with dehydration kinetics of $-0.0229 \mathrm{MPa}^{-1}$ and $-0.0480 \mathrm{MPa} . \mathrm{s}^{-1}$ to obtain respectively final water potentials of $-166.2 \mathrm{MPa}$ and $-317.9 \mathrm{MPa}$. Initial water potential: $-1.4 \mathrm{MPa}$.

Température de réhydratation

\begin{tabular}{lll}
\cline { 2 - 3 } & $25^{\circ} \mathrm{C}$ & $37^{\circ} \mathrm{C}$ \\
\hline$\psi$ final $=-166,2 \mathrm{MPa}$ & $38 \pm 4$ & $59 \pm 8$ \\
$\psi$ final $=-317,9 \mathrm{MPa}$ & $35 \pm 4$ & $61 \pm 5$ \\
\hline
\end{tabular}

hydrique testés, alors qu'à $25^{\circ} \mathrm{C}$ la viabilité n'est que de 38 et $35 \%$.

\subsubsection{Influence d'un stress nutritionnel}

La préparation des cellules à subir la déshydratation par la réalisation d'un stress nutritionnel associé à une déshydratation selon une cinétique optimale et à une réhydratation à $37^{\circ} \mathrm{C}$ permet de maintenir plus de $80 \%$ de cellules viables (tableau IV).

\subsection{4. Étude de conservation des échantillons déshydratés}

Les résultats réunis dans le tableau $V$ montrent que les échantillons stockés à un potentiel hydrique de $-166,2 \mathrm{MPa}$ se conservent mal. En effet, le pourcentage de viabilité chute très rapidement, il n'y a plus que $37 \%$ de cellules viables après 90 jours de stockage. En revanche, pour les échantillons stockés à un potentiel hydrique de $-317,9 \mathrm{MPa}$, le pourcentage de viabilité reste important après 90 jours de stockage.

Ces résultats montrent l'importance du potentiel hydrique de stockage sur la conservation des échantillons. Plus le potentiel hydrique est faible, meilleure est la conservation.

\subsubsection{Mesure de l'activité acidifiante de L. plantarum après réhydratation en milieu MRS à $37^{\circ} \mathrm{C}$}

Les cinétiques d'acidification de L. plantarum ayant subi différents traite- 
Tableau IV. Effet d'un stress nutritionnel sur la viabilité (\%) de L. plantarum déshydraté par séchage en milieu farine eau selon des cinétiques de déshydratation de $-0,0229 \mathrm{MPa} . \mathrm{s}^{-1}$ et $-0,0480 \mathrm{MPa} . \mathrm{s}^{-1}$ afin d'obtenir respectivement des potentiels hydriques finaux de $-166,2 \mathrm{MPa}$ et $-317,9 \mathrm{MPa}$. Réhydratation à $37^{\circ} \mathrm{C}$. Potentiel hydrique initial $=-1,4 \mathrm{MPa}$.

Table IV. Effect of a nutritional stress on $L$. plantarum viability dehydrated by drying in a flour-water mixture with dehydration kinetics of $-0.0229 \mathrm{MPa} . \mathrm{s}^{-1}$ and $-0.0480 \mathrm{MPa} . \mathrm{s}^{-1}$ to obtain respectively final water potentials of $-166.2 \mathrm{MPa}$ and $-317.9 \mathrm{MPa}$. Rehydration temperature: $37^{\circ} \mathrm{C}$.

\section{Réhydratation à $37^{\circ} \mathrm{C} \quad$ Stress nutritionnel + réhydratation}

$37^{\circ} \mathrm{C}$

$\begin{array}{lll}\psi \text { final }=-166,2 \mathrm{MPa} & 59 \pm 8 & 90 \pm 10 \\ \psi \text { final }=-317,9 \mathrm{MPa} & 61 \pm 5 & 83 \pm 8\end{array}$

Tableau V. Conservation de $L$. plantarum déshydraté par séchage en milieu farine eau selon des cinétiques de déshydratation de $-0,0229 \mathrm{MPa} . \mathrm{s}^{-1}$ et $-0,0480 \mathrm{MPa} . \mathrm{s}^{-1}$ afin d'obtenir respectivement des potentiels hydriques finaux de $-166,2 \mathrm{MPa}$ et $-317,9 \mathrm{MPa}$. Conservation sous azote, à $4{ }^{\circ} \mathrm{C}$, à l'obscurité. Réhydratation à $37^{\circ} \mathrm{C}$.

Table V. Conservation of $L$. plantarum dehydrated by drying in a flour-water mixture with dehydration kinetics of $-0.0229 \mathrm{MPa} . \mathrm{s}^{-1}$ and $-0.0480 \mathrm{MPa}^{-1}$ to obtain respectively final water potentials of $-166.2 \mathrm{MPa}$ and $-317.9 \mathrm{MPa}$. Rehydration temperature: $37^{\circ} \mathrm{C}$. Conservation is realised under nitrogen atmosphere, at $4{ }^{\circ} \mathrm{C}$, in darkness.

\begin{tabular}{llccc}
\hline & \multicolumn{4}{c}{ Temps de conservation (jours) } \\
\cline { 2 - 5 } & \multicolumn{1}{c}{0} & 15 & 30 & 90 \\
\hline$\psi$ final $=-166,2 \mathrm{MPa}$ & $90 \pm 10$ & $58 \pm 3$ & $49 \pm 11$ & $37 \pm 3$ \\
$\psi$ final $=-317,9 \mathrm{MPa}$ & $83 \pm 8$ & $81 \pm 6$ & $77 \pm 7$ & $78 \pm 5$ \\
\hline
\end{tabular}

ments sont représentées sur la figure 1. La phase de latence de l'échantillon déshydraté par séchage est légèrement plus longue que celle de l'échantillon témoin (+98 min). En revanche, elle n'est pas plus longue que celle de l'échantillon lyophilisé et actuellement commercialisé. Par conséquent, le procédé de déshydratation par séchage n'altère que faiblement l'activité acidifiante de la bactérie, ce qui apparaît donc très intéressant. De plus, l'échantillon déshydraté par séchage se montre aussi actif que le produit lyophilisé, ce qui augmente son intérêt.

\section{DISCUSSION}

Il a été mis en évidence au cours de cette étude que la cinétique de variation du potentiel hydrique, c'est-à-dire la cinétique de déshydratation, est un paramètre fondamental qui agit sur la viabilité cellulaire. Des cinétiques lentes de diminution du potentiel hydrique ont permis d'améliorer significativement les viabilités observées lors de diminutions rapides du potentiel hydrique.

Lors de la déshydratation par addition de glycérol, des cinétiques optimales de 
Figure 1. Cinétique d'acidification, en milieu MRS à $30^{\circ} \mathrm{C}$, de Lactobacillus plantarum :

(—) témoin non déshydraté, (-) L. plantarum déshydraté par séchage en milieu farine eau, $(\longrightarrow$ L. plantarum déshydraté par lyophilisation. Réhydratation à $37^{\circ} \mathrm{C}$.

Figure 1. Acidification kinetics of $L$. plantarum in MRS medium at $30{ }^{\circ} \mathrm{C} .(-)$ control, (-) L. plantarum dehydrated by drying in a flour-water mixture, (-) L. plantarum dehydrated by freeze drying. Rehydration temperature: $37^{\circ} \mathrm{C}$.

déshydratation ont été déterminées, permettant de maintenir plus de $80 \%$ des cellules viables. L'existence de ces cinétiques optimales peut être expliquée par l'opposition de deux actions sur la viabilité cellulaire. Premièrement, une action positive qui est la diminution lente du potentiel hydrique et deuxièmement, une action négative qui est le temps de résidence des cellules dans une zone critique de potentiel hydrique [8].

Ces résultats peuvent être comparés avec ceux obtenus par Gervais et al. [4] et Marechal et Gervais [7] sur les levures. Ces auteurs ont montré l'influence de la vitesse de diminution du potentiel hydrique sur la survie de deux levures: Saccharomyces cerevisiae et Sporodiobolus salmonicolor.

Lors d'une diminution du potentiel hydrique du milieu extracellulaire, la sortie d'eau des cellules est proportionnelle au gradient de potentiel hydrique de part et d'autre de la membrane. Lors d'un choc, ce gradient est important et instantané. En revanche, des cinétiques lentes de variation du potentiel hydrique entraînent de faibles

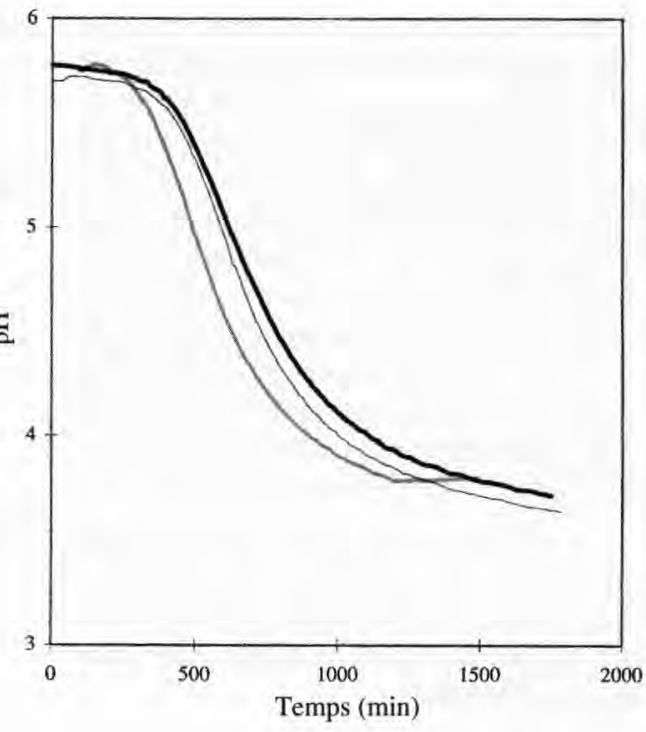

gradients de potentiel hydrique entre les milieux intra- et extracellulaire. Dans ce cas, les flux d'eau traversant la membrane sont de faible intensité et n'entrainent pas d'altérations membranaires.

La zone critique de potentiels hydriques $(-170,9 \mathrm{MPa} \sim-244,7 \mathrm{MPa})$ peut être expliquée par l'existence, à ce niveau, d'une transition de phase induite par la déshydratation.

La transposition des cinétiques de déshydratation en milieu liquide à des cinétiques de déshydratation par séchage (air sec à basse température) a permis de confirmer les résultats précédents. Des cinétiques optimales de déshydratation par séchage ont été déterminées, permettant de maintenir $40 \%$ de cellules viables.

La déshydratation par séchage a également permis de mettre en évidence l'importance de la température de réhydratation sur la viabilité cellulaire ainsi que la possibilité de prévenir la mortalité des bactéries par l'application d'un stress nutritionnel avant la déshydratation. Cette adaptation permet de mettre en évidence la similitude des réponses des microorga- 
nismes face aux stress. D'autre part, il apparaît qu'il existe une température optimale de réhydratation. Il conviendrait de tester des températures supérieures à celles utilisées dans notre étude $\left(25^{\circ} \mathrm{C}, 37^{\circ} \mathrm{C}\right)$, par exemple $50^{\circ} \mathrm{C}$. En effet, Teixeira et al. [9] ont montré que la température optimale de réhydratation de Lactobacillus bulgaricus déshydratée par séchage est de $50^{\circ} \mathrm{C}$. L'élévation de température lors de la réhydratation permettrait d'augmenter la fluidité membranaire, rendant la membrane plus apte à retrouver un état hydraté [6].

$\mathrm{La}$ réalisation du stress nutritionnel, quant à lui, va permettre aux bactéries d'accumuler des substances de réserves qui leur permettront de mieux supporter la déshydratation.

Cette étude nous a permis de déterminer les conditions optimales permettant de déshydrater $L$. plantarum par séchage avec de l'air sec à basse température, en maintenant plus de $80 \%$ de viabilité. De plus, l'étude de conservation des produits déshydratés montre l'importance du potentiel hydrique de stockage sur le maintien de la viabilité cellulaire. Un potentiel hydrique de $-317,9 \mathrm{MPa}$ permet de maintenir près de $80 \%$ de cellules viables sur une période de trois mois. D'autres études montrent également l'importance du potentiel hydrique de stockage sur la conservation des produits déshydratés. Ainsi, Lactobacillus delbrueckii ssp. bulgaricus présente une conservation optimale à un potentiel hydrique de $-304,8 \mathrm{MPa}$ [9]. Enfin, notre procédé de déshydratation par séchage n'altère pas de façon importante l'activité acidifiante de $L$. plantarum. La phase de latence de la cinétique d'acidification du produit déshydraté par notre procédé est peu différente de celle du témoin non déshydraté et identique à celle du produit lyophilisé.

Par ailleurs, la méthode de déshydratation proposée dans cette étude n'utilise pas de solutés protecteurs, contrairement à la lyophilisation, ce qui permet de dimi- nuer le coût du produit et d'élargir son domaine d'application.

\section{REMERCIEMENTS}

Cette étude a été soutenue financièrement par la société Eurogerm et par le Conseil régional de Bourgogne.

\section{RÉFÉRENCES}

[1] Becker M.J., Rapoport A.I., Conservation of yeasts by dehydration, Adv. Biochem. Eng. Biotechnol. 35 (1987) 127-171.

[2] Brown A.D., Edgley M., Osmoregulation in yeast, in: Rains D.W., Valentine R.C., Hollaender A. (Eds.), Genetic Engineering of Osmoregulation, Plenum Press, New-York, 1980, pp. 75-90.

[3] De Man J.C., Rogosa M., Sharpe M.E., A medium for the cultivation of Lactobacilli, J. Appl. Bacteriol. 23 (1960) 130-135.

[4] Gervais P., Marechal P.A., Molin P., Effects of the kinetics of osmotic pressure variation on yeast viability, Biotechnol. Bioeng. 43 (1992) $165-170$.

[5] Leslie L.B., Israeli, Lighthart B., Crowe J.H., Crowe L.M., Trehalose and sucrose protect both membranes and proteins in intact bacteria during drying, Appl. Environ. Microbiol. 61 (1995) 3592-3597.

[6] Macdonald A.G., Wahle K.W.J., Cossins A.R., Behan M.K., Temperature, pressure and cholesterol effects on bilayer fluidity; a comparison of pyrene excimer/monomer ratios with the steady-state fluorescence polarization of diphenylhexatriene in liposomes and microsomes, Biochem. Biophys. Acta 938 (1988) 231-242.

[7] Marechal P,A., Gervais P., Yeast viability related to water potential variation: influence of the transient phase, Appl. Microbiol. Biotechnol. 42 (1994) 617-622.

[8] Poirier I., Marechal P.A., Gervais P., Effects of the kinetics of water potential variation on bacteria viability, J. Appl. Bacteriol. 82 (1997) 101-106.

[9] Teixeira P.C., Castro M.H., Malcata F.X., Kirby R.M., Survival of Lactobacillus delbrueckii ssp. bulgaricus following spraydrying, J. Dairy Sci. 78 (1995) 1025-1031. 